\title{
Ann Jefferson, Biography and the Question of Literature in France
}

\section{Luigi Luison}

\section{(2) OpenEdition}

1 Journals

\section{Edizione digitale}

URL: http://journals.openedition.org/studifrancesi/9076

DOI: 10.4000/studifrancesi.9076

ISSN: 2421-5856

\section{Editore}

Rosenberg \& Sellier

\section{Edizione cartacea}

Data di pubblicazione: 1 octobre 2008

Paginazione: 504-505

ISSN: 0039-2944

\section{Notizia bibliografica digitale}

Luigi Luison, «Ann Jefferson, Biography and the Question of Literature in France», Studi Francesi [Online], 155 (LII | II) | 2008, online dal 30 novembre 2015, consultato il 13 janvier 2021. URL: http:// journals.openedition.org/studifrancesi/9076 ; DOI: https://doi.org/10.4000/studifrancesi.9076

Questo documento è stato generato automaticamente il 13 janvier 2021.

\section{(c) (i) $\odot$}

Studi Francesi è distribuita con Licenza Creative Commons Attribuzione - Non commerciale - Non opere derivate 4.0 Internazionale. 


\title{
Ann Jefferson, Biography and the Question of Literature in France
}

\author{
Luigi Luison
}

\section{NOTIZIA}

ANN JEFFERSON, Biography and the Question of Literature in France, Oxford University Press, 2007, pp. 425.

1 Come punto di partenza per il suo saggio, che studia il rapporto tra letteratura e biografia, Ann JEFFERSON prende in esame il valore apparente dei due termini "letteratura" (intesa nel senso moderno) e "biografia", tenendo conto di alcune possibili implicazioni, come il fatto che l'esigenza della "letteratura" sia in qualche modo legata ad uno strategico uso della biografia, e che la nozione stessa di letteratura sia aperta ad una contestazione che risale indietro nei secoli. Lo scopo di questo lavoro, circoscritto alla letteratura francese, è di mettere in evidenza le varie forme assunte dalla relazione tra letteratura e biografia, relazione che non può essere ridotta ad una singola formula o una semplice presupposizione. Il volume si divide in otto parti e presenta un percorso che va da Rousseau ai giorni nostri.

2 La prima parte (“Lives" and the invention of "Literature"», pp. 31-78) mira a identificare, dalla fine del xvII secolo, un'essenza letteraria o poetica che trascenda la varietà di forme e generi differenti. I tre capitoli di questa prima parte espongono le diverse strutture emerse dalle reciproche relazioni tra biografia e letteratura prima delle loro totali manifestazioni nel XIX secolo. Le Confessions di Rousseau è il primo testo ad invocare una nuova idea di letteratura, e in questo modo apre esplicitamente l'intero campo letterario ad interrogativi e contestazioni. Nel 1778 il critico LouisSébastien Mercier pubblica un saggio dal titolo De la littérature et des littératures, con il quale argomenta sul fatto che la letteratura e le belles lettres abbiano il potere di promuovere la virtù ed elevare la mente. Egli attribuisce questa qualità alla sensibilità morale degli scrittori, piuttosto che ad altre caratteristiche formali di poesia, dramma o 
racconto, i cui effetti, peraltro, egli difende. In questa discussione, il contributo più significativo è dato dal testo di Madame de Staël, De la littérature, del 1800, con cui la scrittrice include nella letteratura poesia, eloquenza, storia e filosofia. Nonostante l'ampiezza della sua definizione, Madame de Staël tratta la nozione di letteratura come un'unica entità, esaminata attraverso le sue relazioni con il contesto sociale. In pratica, l'estensione del termine "letteratura", emerso nella seconda metà del xviII secolo, associa il concetto a quello di missione (nel caso di Mercier) o a quello di storia (nel caso del Lycée di La Harpe) o, nel caso di Madame de Staël, ad entrambi. Inoltre, volta al passato (la sua storia) o al futuro (la sua missione), la letteratura ora sembra dipendere da una temporalità dinamica in cui la storia letteraria acquista il carattere di una narrativa biografica. Con la Préface de Cromwell del 1827, Victor Hugo adotta un modello organicistico più completo di quello di Madame de Staël; un modello che rappresenta il topos di una storia biologica dell'umanità o della società e che diventerà molto più comune durante la prima metà del XIX secolo.

3 La seconda parte («The 19th-Century culture of biography», pp. 83-134) prende in esame gli ultimi anni del XIX secolo, contrassegnati da un'improvvisa intensificazione della scrittura biografica. In particolare, il ritratto letterario vede, sia nella versione giornalistica che in quella sotto forma di raccolta, un particolare rinnovamento polemico. In questo contesto si inseriscono gli articoli di Verlaine su Les hommes d'aujourd'hui, la serie pubblicata da Léon Vanier, oltre a quelli di Anatole France, Barbey d'Aurevilly, Villiers de l'Isle-Adam e Mallarmé. Non va dimenticato, inoltre, il Livre des masques di Rémy de Gourmont del 1896 che include una schiera di giovani scrittori come Rachilde, Lautréamont, Henri de Régnier, André Gide e Marcel Schwob. In questo periodo, la giustificazione per un ritratto biografico risiede nella supposizione che un lavoro letterario sia il riflesso della personalità individuale dello scrittore. Individualità e originalità diventano così i criteri per valutare un lavoro letterario che pone in essere una nuova estetica, espressa inevitabilmente in ogni nuovo ritratto. Ed è proprio la questione dell'estetica letteraria ad essere il nucleo della collezione di ritratti biografici di Mallarmé, Quelques médaillons et portraits en pied del 1897. La forma biografica utilizzata da Mallarmé è quella del necrologio, piuttosto che la biografia contemporanea più comunemente presente nella stampa del tempo. Ma in aggiunta a tutto ciò, il XIX secolo vede anche la nascita della critica biografica, il cui maggior esponente è Sainte-Beuve, per il quale la critica biografica non è solo una forma di critica tra le tante altre esistenti, ma, in generale, il pretesto per una completa trasformazione della cronaca letteraria nella sua forma moderna.

Nella terza parte («Poetry and the life of the poet», pp. 143-180) l'A. prende in esame le figure di Hugo e Baudelaire: a metà Ottocento, con i due scrittori la questione della biografia si muove decisamente verso l'ambito poetico. Ne Les Contemplations di Hugo e Les Fleurs du mal di Baudelaire la biografia è incorporata nella stoffa del lavoro. Le due opere hanno in comune, rileva l'A., due elementi significativi: in ognuna, la biografia del poeta non è usata come semplice sfondo o prefazione, ma come una principale struttura attiva per l'insieme della collezione poetica; e questa struttura biografica è usata per ragioni di ridefinizione e promozione dell'idea della poesia stessa. Nella quarta parte («Biography into literature», pp. 185-217) sono poi analizzati Les Illuminés di Nerval e le Vies imaginaires di Marcel Schwob. Dove Schwob rende contingente il particolare biografico in un principio estetico centrale, Nerval giunge sulla questione più indirettamente, individuando i procedimenti biografici e mettendoli in primo piano 
nella sua narrativa allo scopo di sollevare interrogativi sul potenziale stesso della letteratura. Se le Vies imaginaires di Schwob si prestano ad esser lette come letteratura, Les illuminés di Nerval si collocano più ambiguamente tra l'ambito del ritratto critico, la biografia storica e le considerazioni su un progetto letterario.

5 Nella quinta parte («Inwardness, experience, and the turn to fiction», pp. 223-264) vengono prese in esame le prime decadi del Novecento. In questi anni la biografia comincia ad acquisire per la prima volta lo status di genere, considerato come una forma di espressione letteraria al pari del romanzo. Il nuovo fenomeno della cosiddetta vie romancée viene visto, se letto positivamente, come un modo per mettere le tecniche narrative del racconto al servizio della biografia; letto negativamente, invece, viene considerato come un sottoprodotto della narrativa di finzione. Il dibattito porterà a interventi importanti sulle forme e le pratiche della narrativa biografica, culminanti nell'opera di André Gide, Aspects de la biographie del 1928. Secondo Ann Jefferson, però, l'annessione della biografia alla letteratura non procede dal genere delle vies romancées, bensì da testi quali l'autobiografia finzionale di Proust o Si le grain ne meurt di André Gide.

6 Nel corso del xx secolo, la maggior preoccupazione viene a essere quella del processo creativo. Ma laddove per Proust e Gide il punto focale è in gran parte sull'interiorità di questa esperienza, Leiris e Sartre, circa vent'anni più tardi, intendono la creazione soprattutto come una forma d'azione dalle conseguenze non solo interiori ma anche esterne. Questo è l'argomento trattato nella sesta parte («Acts of literature: the sacred and the writer's life», pp. 269-305): la biografia e l'autobiografia, come spiega Leiris, diventano la struttura dentro la quale il valore della letteratura può essere meglio misurato. La nozione di "azione autentica" è in effetti centrale sia in Leiris che in Sartre, ma i due scrittori differiscono radicalmente nel modo in cui considerano lo status della letteratura. Il progetto di Leiris è volto a risacralizzare il letterario, mentre il fine di Sartre è una totale desacralizzazione della missione della letteratura. Secondo la visione sartriana la letteratura, scelta esistenziale fatta dallo scrittore, espone al rischio dell'inautenticità; per Leiris, invece, la valorizzazione della letteratura si può ottenere solo se lo scrittore pone se stesso in una posizione dove la sua attività potrebbe richiedergli di pagare con la vita.

7 Nella settima parte («Heroes, saints, and generic anachronisms», pp. 311-351) l'A. illustra un cambio di direzione. Genet (che scrive tra gli anni Quaranta e Cinquanta) e Pierre Michon (che scrive tra gli anni Ottanta e Novanta) scelgono di presentare se stessi come scrittori di "vite", piuttosto che come "biografi". Genet, generalmente considerato autore autobiografico, mira in realtà a celebrare le vite dei suoi eroisoggetti, e ricorre a modelli di scrittura medievali. Pierre Michon si impegna in un tipo simile di sperimentazione generica, e le sue ambizioni letterarie sono realizzate in una riesumazione della forma delle vite dei santi. "Agiografia" è un termine usato spesso in accezione denigratoria, ma adottando il modello della vita dei santi, Michon rivisita radicalmente le ipotesi della scrittura biografica contemporanea in modi che, come le "vite" degli eroi di Genet, pongono l'elemento letterario al centro dei suoi interessi.

8 L'ottava e ultima parte («The writing life», pp. 357-386) tratta dell'arte come vocazione: la questione della natura e la funzione dell'arte è stata spesso associata, nei secoli passati, alla nozione di vocazione dello scrittore. Quando l'arte diventa una vocazione (una chiamata alle cose più elevate, secondo il pensiero di Hölderlin) crea uno speciale modo di vita per il poeta, che lo pone rispetto ai suoi simili alla stessa distanza che 
separa gli uomini dagli animali. Questo tipo di vocazione e il "regime" delineato da George Eliot, sono i principali indicatori della vita di un artista. È in questa prospettiva che la vita dell'artista viene intesa come la vera fonte della creazione artistica. L'A. procede, in conclusione, ad analizzare la personificazione dei problemi di vocazione e regime rispetto alle figure di due scrittori francesi contemporanei, Roger Laporte e Jacques Roubaud. 